\title{
Glucocorticoid treatment is associated with ICU-acquired hypernatremia: a nested case-control study
}

\author{
Takahiro Imaizumi ${ }^{1} \cdot$ Masahiro Nakatochi $^{2} \cdot$ Yoshiro Fujita $^{3} \cdot$ Rie Yamamoto $^{4} \cdot$ Kennshi Watanabe $^{4}$. \\ Michitaka Maekawa ${ }^{4} \cdot$ Taishi Yamawaka $^{4} \cdot$ Takayuki Katsuno $^{5} \cdot$ Shoichi Maruyama ${ }^{1}$
}

Received: 1 July 2020 / Accepted: 2 September 2020 / Published online: 22 September 2020

(C) Japanese Society of Nephrology 2020

\begin{abstract}
Background Hypernatremia is a major electrolyte disorder associated with death among critically ill patients. Glucocorticoid therapy may cause hypernatremia in refractory septic shock patients, but the association between glucocorticoid and intensive care unit (ICU)-acquired hypernatremia (IAH) remains unclear. The aim of this study was to clarify whether glucocorticoid administration was associated with IAH.

Methods This was a nested case-control study using data from an established cohort including 121 IAH cases identified from 1756 patients who were admitted to ICU in a tertiary care facility in Japan. We included patients who were admitted with a normal range of serum sodium concentrations (130-149 mEq/L) from January 1, 2013 to December 31, 2015 and remained in ICU for $\geq 2$ days. Hypernatremia was defined as serum sodium concentration $\geq 150 \mathrm{mEq} / \mathrm{L}$. Each case was matched to one control.

Results Multivariable conditional logistic regression revealed high-dose glucocorticoid \{odds ratio (OR), 4.15 [95\% confidence interval (CI) 1.29-13.4]\}, acute kidney injury (AKI) [OR, 2.72 (95\% CI 1.31-5.62)], and osmotic diuretics [OR, 3.44 (95\% CI 1.41-8.39)] to be significantly associated with IAH. The contents and amounts of fluid infusion were not significantly associated with IAH. There were also significant duration-response effects between duration of glucocorticoid use and IAH; however, pulse glucocorticoid administration was not associated with IAH.

Conclusion In this nested case-control study, we demonstrated a significant association between IAH and high-dose glucocorticoid with significant duration-response effects. Serum sodium concentrations should be monitored carefully in critically ill patients administered prolonged high-dose glucocorticoid.
\end{abstract}

Keywords Hypernatremia $\cdot$ Intensive care unit · Glucocorticoid · Epidemiology $\cdot$ Nested case-control study $\cdot$ Incidence density sampling

Electronic supplementary material The online version of this article (https://doi.org/10.1007/s10157-020-01967-9) contains supplementary material, which is available to authorized users.

Shoichi Maruyama

marus@med.nagoya-u.ac.jp

1 Department of Nephrology, Nagoya University Graduate School of Medicine, 65 Tsurumai-cho, Showa-ku, Nagoya 466-8550, Japan

2 Biostatistics and Bioinformatics Section, Center for Advanced Medicine and Clinical Research, Nagoya University Hospital, Nagoya, Japan

\section{Introduction}

Hypernatremia is one of the major electrolyte disorders associated with death among critically ill patients. Previous studies have shown that intensive care unit-acquired hypernatremia (IAH) was an independent predictor of increased

3 Department of Nephrology, Chubu Rosai Hospital, Nagoya, Japan

4 Department of Nephrology, Toyohashi Municipal Hospital, Toyohashi, Japan

5 Department of Nephrology and Rheumatology, Aichi Medical University, Nagakute, Japan 
mortality [1-5]. Hypernatremia causes many adverse effects such as neurologic dysfunction and decreased left ventricular contractility that might lead to prolonged duration of mechanical ventilation and delayed weaning [6-8]. Thus, the prevention of hypernatremia in critically ill patients is crucial.

Hypernatremia is caused by electrolyte-free water depletion resulting from defective urinary concentration or increased insensible and enteral losses, in addition to inadequate water intake or fluid management $[9,10]$. Previous studies have revealed that mannitol, renal insufficiency, mechanical ventilation, sepsis, hypokalemia, and sodium bicarbonate are the risk factors for IAH [2, 4]. Insensible water loss is caused by hyperventilation, high body temperature, or under mechanical ventilation. Urinary concentrating defects are caused by impaired water reabsorption in the collecting duct and by osmotic diuresis.

Osmotic diuresis can lead to a significant loss of electrolyte-free water and is caused by osmotic diuretics such as mannitol and glycerol, glucose, and urea. Osmotic diuresis is also caused by urea, and it can be observed in patients with sepsis, respiratory failure, and other catabolic states [11].

Glucocorticoid treatment leads to an increase in the levels of blood urea nitrogen (BUN) via catabolism. Overproduction of BUN plays an important role in osmotic diuresis. A systematic review revealed that glucocorticoid therapy was associated with hypernatremia among patients with refractory septic shock [12]. Another retrospective cohort study showed that steroid therapy was associated with intensive care unit (ICU)-acquired hypernatremia (IAH), although the authors did not provided any definition, duration, or dosages of steroid treatment involved, including whether or not pulse treatment of glucocorticoids was administered [4]. Thus, the relation between glucocorticoid and IAH has largely remained unclear.

Herein, we aimed to examine whether glucocorticoid administration was associated with IAH using a nested case-control study design, which can minimize the selection bias.

\section{Methods}

\section{Study population, setting, and definitions}

This study was a nested case-control study using data from an established cohort. The cohort was established for the purpose of exploring electrolyte disorders in patients admitted to the ICU of a tertiary referral hospital from January 1, 2013 through December 31, 2015 [13]. The cohort included 1756 adult patients ( $\geq 18$ years old) whose length of stay in the ICU was $>2$ days, and whose serum sodium concentrations were $130-149 \mathrm{mEq} / \mathrm{L}$ on ICU admission. We excluded patients whose initial sodium concentration results were missing and brain-dead patients admitted to the ICU for planed organ donation. The ICU at our institution manages both medical and surgical ICU patients and is staffed by non-intensivists.

If a patient was admitted to the ICU more than once during a hospital admission, data were obtained from the first ICU admission that was longer than 2 days in duration or from the admission during which the patient developed IAH. Patients who developed hypernatremia outside of the ICU were not classified as IAH.

Hypernatremia was defined as serum sodium concentration $\geq 150 \mathrm{mEq} / \mathrm{L}$. We defined that IAH as hypernatremia occurring $\geq 12 \mathrm{~h}$ after ICU admission in patients with normal serum sodium at ICU admission. Patients who developed hypernatremia within the first $12 \mathrm{~h}$ of ICU admission were excluded. Glucocorticoids administered in this study included prednisolone, methylprednisolone, dexamethasone, betamethasone, and hydrocortisone. According to the report of the European League Against Rheumatism, high-dose glucocorticoid is defined as $>30 \mathrm{mg}$ prednisone equivalent per day, and pulse therapy consists of $>250 \mathrm{mg}$ prednisone equivalent per day for one or a few days [14]. We considered the cumulative amount and duration of glucocorticoid as administered in ICU before developing IAH.

\section{Study design}

In this study, we used a nested case-control design in which the controls were sampled from the risk set by incidence density sampling [15]. The date from ICU admission to IAH diagnosis for each case was the index date. The same index date from ICU admission for each case was assigned to each of the matched controls, which had to be alive, have stayed in the ICU, and had not developed IAH, to ensure similar follow-up times between cases and controls. Using this approach, the risk set of a given case consisted of all subjects at risk. By this definition, another case was a potential control if he developed IAH in later date. We sampled one control within each risk set. We used the Stata software and the "sttocc" procedure to implement these steps [16].

\section{Data collection}

The following data were recorded: diagnosis on ICU admission, age, sex, vital signs, the Glasgow Coma Scale (GCS) score, and the Acute Physiology And Chronic Health Evaluation II (APACHEII) score [17] on the ICU admission; biochemical variables; ICU exposures such as mechanical ventilation or renal replacement therapy; complications developed in ICU, such as sepsis or acute kidney injury (AKI); medications including glucocorticoid; and the contents of infusion fluid in ICU. The main diagnosis on ICU 
admission was classified into the following categories: cardiovascular disease, gastrointestinal disease, neurological disease sepsis, respiratory disease, and others. Cardiovascular disease included heart diseases, post-cardiac surgery, and vascular diseases other than cerebrovascular disease. The neurological disease category included cerebrovascular disease, traumatic head injury, refractory status epilepticus, and encephalopathy.

Sepsis is defined as life-threatening organ dysfunction caused by a dysregulated host response to infection. Organ dysfunction was represented by an increase in the Sepsisrelated Organ Failure Assessment (SOFA) score of 2 points or more [18]. AKI was defined in accordance with the Kidney Disease: Improving Global Outcome guidelines [19].

Parameters were recorded in cases in the period between the ICU admission and the first date of hypernatremia in the ICU, and in controls using an equivalent time period. Electrolytes and nutrients of the infusion fluid (such as sodium, potassium, glucose, and amino acids) were calculated from chart data obtained $24 \mathrm{~h}$ before development of hypernatremia. For patients who developed hypernatremia on the day following admission to ICU, data were obtained from the previous $12 \mathrm{~h}$, and the values doubled. Urine output was calculated similarly. Infusion fluid contained the fluid from both parenteral and enteric nutrition, but did not included elements taken orally.

\section{Statistical analysis}

Clinical characteristics were compared between the IAH and non-IAH cases using the Wilcoxon signed-rank test for continuous variables and the McNemar Chi-squared test or exact McNemar's test for categorical variables, as appropriate. Continuous variables were expressed as median [interquartile range (IQR)], and categorical variables were expressed as number and proportion, as appropriate.

To identify predictors of IAH, cases were compared to controls using conditional logistic regression analysis to allow a comparison in the same matched set. Potential covariates and confounders with IAH were identified using clinical knowledge and data from the literature [2, 4]. We performed two multivariable analyses. Model 1 was adjusted for age, gender, APACHEII score, sepsis, AKI, use of osmotic diuretics, use of loop diuretics, use of high-dose glucocorticoid, use of a mechanical ventilator, initiation of renal replacement therapy, and emergency operations prior to admission. Model 2 was adjusted for the same variables as Model 1, plus the amount and the contents of infusion fluid present the day before developing IAH.

We also examined dose-response and duration-response effects of glucocorticoid on IAH. Here, we defined "dose" as the presence or absence of pulse glucocorticoid administration, because cumulative doses of glucocorticoid were dependent on the presence or absence of pulse administration. We did not examine the effects of the total amount or initial dosage of glucocorticoid. We also defined "duration" as the duration of glucocorticoid administration from ICU admission to the development of IAH. We examined the duration of glucocorticoid as both continuous and categorical variables, which were classified as no use, $\geq 1$ to $<3$ days, $\geq 4$ to $<6$ days, and $\geq 7$ days. We considered those not administered any glucocorticoids as the reference category. The trend in the outcome with respect to the duration of glucocorticoid administration was examined statistically by scoring no use as 0 and glucocorticoid-administration with $1-3,4-6$, and 7 days or more as 1,2 , and 3 , respectively; the resulting scores were then included in the regression model.

Finally, we also compared the differences in clinical characteristics and contents of fluid infusion between IAH cases associated with glucocorticoid and those without.

The statistical significance was set at $p<0.05$. All statistical analyses were conducted using Stata version 14.1 (Stata Corp., College Station, TX, USA; https://www.stata.com).

\section{Results}

\section{Characteristics of cases and controls}

A total of $121 \mathrm{IAH}$ cases and 121 controls were extracted from the cohort to construct this nested case-control study. Baseline characteristics, treatment exposures, complications, and outcomes in ICU are summarized in Table 1. ICU treatment exposures and complications were obtained from the data before the date of IAH development in cases and from the equivalent date in controls. Baseline levels of the APACHEII score (median 19 versus 16, $p=0.0053$ ), BUN (median 23 versus $19 \mathrm{mg} / \mathrm{dL}, p=0.0020$ ) and CRP (median 2.43 versus $0.70 \mathrm{mg} / \mathrm{dL}, p=0.014$ ) were significantly higher, while the GCS scale was significantly lower among IAH cases (median 12 versus 15, $p<0.001$ ). Baseline levels of serum sodium concentration were not significantly different between IAH cases and controls (median 142 versus $141 \mathrm{mEq} / \mathrm{L}, p=0.17)$. AKI and administration of tolvaptan were significantly more frequent in cases compared to controls $(66.1 \%$ versus $43.0 \%, p=0.0011,3.3 \%$ versus $0 \%$, $p=0.046$, respectively). The frequency of administration of osmotic diuretics was not significantly higher in cases compared to controls (36.4\% versus $25.6 \%, p=0.069)$ and as was for loop diuretics (40.5\% versus $30.6 \%, p=0.090)$, but was higher among IAH case administration of high-dose glucocorticoid was significantly more frequent in IAH cases compared to controls ( $19 \%$ versus $9.9 \%, p=0.034$ ), and the duration of glucocorticoid administration was significantly longer (median 5 versus 3 days, $p=0.014$ ). However, the 
Table 1 Baseline characteristics, complications, exposures, and interventions in ICU and outcomes

\begin{tabular}{|c|c|c|c|c|}
\hline Category & Variable & Cases $(n=121)$ & Controls $(n=121)$ & $p$ value \\
\hline \multirow[t]{6}{*}{ Diagnosis on admission } & Cardiovascular disease & $16(13.2)$ & $29(24.0)$ & 0.060 \\
\hline & Gastrointestinal disease & $5(4.1)$ & $7(5.8)$ & 0.75 \\
\hline & Neurological disease & $45(37.2)$ & $36(29.8)$ & 0.24 \\
\hline & Respiratory disease & $13(10.7)$ & $16(13.2)$ & 0.69 \\
\hline & Sepsis & $29(24.0)$ & $16(13.2)$ & $0.041^{\mathrm{c}}$ \\
\hline & $>$ Others & $13(10.7)$ & $17(14.1)$ & 0.57 \\
\hline \multirow[t]{10}{*}{ Baseline characteristics } & Age, years & $71(61,78)$ & $70(60,77)$ & 0.29 \\
\hline & Male, $n(\%)$ & $85(70.3)$ & $79(65.3)$ & 0.37 \\
\hline & Dementia, $n(\%)$ & $14(11.6)$ & $12(9.9)$ & 0.68 \\
\hline & Glasgow Coma Scale score & $12(4,15)$ & $15(11,15)$ & $<0.001^{\mathrm{C}}$ \\
\hline & APACHEII score & $19(13,24)$ & $16(11,22)$ & $0.0053^{c}$ \\
\hline & BMI, $\mathrm{kg} / \mathrm{m}^{2}$ & $20.1(17.7,23.5)$ & $21.2(18.2,23.1)$ & 0.96 \\
\hline & $\mathrm{BUN}, \mathrm{mg} / \mathrm{dL}$ & $23(15,45)$ & $19(13,28)$ & $0.0020^{c}$ \\
\hline & $\mathrm{Cr}, \mathrm{mg} / \mathrm{dL}$ & $1.05(0.72,1.77)$ & $0.94(0.69,1.4)$ & 0.23 \\
\hline & $\mathrm{Na}, \mathrm{mEq} / \mathrm{L}$ & $142(138,144)$ & $141(139,143)$ & 0.17 \\
\hline & $\mathrm{CRP}, \mathrm{mg} / \mathrm{dL}$ & $2.43(0.15,12.9)$ & $0.70(0.09,6.5)$ & $0.014^{\mathrm{c}}$ \\
\hline \multirow[t]{3}{*}{ Complications $^{\mathrm{a}}$} & AKI, $n(\%)$ & $80(66.1)$ & $52(43.0)$ & $0.0011^{\mathrm{c}}$ \\
\hline & GI bleeding, $n(\%)$ & $7(5.8)$ & $5(4.1)$ & 0.56 \\
\hline & Sepsis, $n(\%)$ & $45(37.2)$ & $33(27.3)$ & 0.096 \\
\hline \multirow[t]{3}{*}{ Interventions ${ }^{\mathrm{a}}$} & Renal replacement therapy, $n(\%)$ & $9(7.4)$ & $17(14.1)$ & 0.088 \\
\hline & Mechanical ventilation, $n(\%)$ & $86(71.1)$ & $79(65.3)$ & 0.26 \\
\hline & Emergency surgery, n (\%) & $27(22.3)$ & $24(19.8)$ & 0.61 \\
\hline \multirow[t]{7}{*}{ Glucocorticoid $^{\mathrm{a}}$} & High-dose glucocorticoid, $n(\%)$ & $23(19.0)$ & $12(9.9)$ & $0.034^{\mathrm{c}}$ \\
\hline & Pulse administration, $n(\%)$ & $5(4.1)$ & $7(5.8)$ & 0.77 \\
\hline & Duration of administration, days & $5(3,8)$ & $3(3,7)$ & $0.014^{\mathrm{c}}$ \\
\hline & Duration, category & & & 0.052 \\
\hline & $1-3$ days & $8(34.8)$ & $7(58.3)$ & \\
\hline & 4-6 days & $5(21.7)$ & $2(16.7)$ & \\
\hline & 7 days- & $10(43.5)$ & $3(25)$ & \\
\hline Medication other than & Osmotic diuretics, $n(\%)$ & $44(36.4)$ & $31(25.6)$ & 0.069 \\
\hline \multirow[t]{3}{*}{ Glucocorticoid $^{\mathrm{a}}$} & Loop diuretics, $n(\%)$ & $49(40.5)$ & $37(30.6)$ & 0.090 \\
\hline & Torvaptan, $n(\%)$ & $4(3.3)$ & $0(0)$ & $0.046^{\mathrm{c}}$ \\
\hline & Sodium bicarbonate, $n(\%)$ & $13(10.7)$ & $7(5.8)$ & 0.16 \\
\hline \multirow[t]{6}{*}{ Electrolytes and nutrients ${ }^{\mathrm{b}}$} & Infusion fluid, L/day & $2.20(1.50,3.00)$ & $2.0(1.25,2.80)$ & $0.031^{\mathrm{c}}$ \\
\hline & Urine output, L/day & $1.89(1.17,2.80)$ & $1.39(0.72,2.10)$ & $0.001^{\mathrm{c}}$ \\
\hline & Sodium, mEq/day & $172.9(77,297)$ & $119.4(55,218)$ & $<0.001^{\mathrm{c}}$ \\
\hline & Potassium, mEq/day & $9.5(0,20)$ & $14.4(3.6,21)$ & 0.17 \\
\hline & Glucose, g/day & $34.4(2.5,133)$ & $28.6(11.7,117)$ & 0.63 \\
\hline & Nitrogen, g/day & $0(0,4.7)$ & $0(0,4.7)$ & 0.59 \\
\hline \multirow[t]{2}{*}{ Outcomes } & Length of ICU, days & $9(5,15)$ & $7(4,15)$ & 0.10 \\
\hline & 28 day mortality, $n(\%)$ & $42(35.0)$ & $17(14.1)$ & $<0.001^{\mathrm{c}}$ \\
\hline
\end{tabular}

Continuous data are median (IQR). Categorical data are $n$ values (\%)

$B M I$ body mass index, APACHE Acute Physiology And Chronic Health Evaluation, AKI acute kidney injury, GI gastrointestinal, ICU intensive care unit

${ }^{a}$ From ICU admission to the day before IAH

${ }^{\mathrm{b}}$ Administered $24 \mathrm{~h}$ before IAH

${ }^{\mathrm{c}} p<0.05$ 
frequency of pulse steroid administration was not statistically different between IAH cases and controls (4.1\% versus $5.8 \%, p=0.77)$.

In the analysis of urine output and the contents of infusion fluid $24 \mathrm{~h}$ before IAH development, patients with IAH had more urine output (median 1.89 versus $1.39 \mathrm{~L} /$ day, $p=0.001$ ) and were administered higher amounts of infusions (median 2.20 versus $2.00 \mathrm{~L} /$ day, $p=0.031$ ) and sodium intake (median 172.9 versus $119.4 \mathrm{mEq} / \mathrm{day}, p<0.001$ ), while there were no statistically significant differences in the amount of glucose and nitrogen between cases and controls.

\section{Risk factors of intensive care unit-acquired hypernatremia}

The results of univariate and multivariable conditional logistic regression analysis are shown in Table 2 . In the multivariable analysis, AKI, the use of high-dose glucocorticoid, and the use of osmotic diuretics were independently significant factors associated with IAH. While the odds ratio (OR) of AKI was significantly high, that of renal replacement therapy was significantly low. Sepsis was not significantly associated with IAH.

\section{Dose-response association between glucocorticoid administration and intensive care unit-acquired hypernatremia}

Table 3 shows that the duration of glucocorticoid administration had a strong independent effect on the risk of IAH. Multivariable conditional logistic regression analysis showed a significant association between the outcome and the duration of glucocorticoid administration expressed as a continuous value $\{\mathrm{OR}, 1.21[95 \%$ confidence $(\mathrm{CI})$ $1.01-1.45], p=0.039\}$, and as a categorical value ( $p$-value for trend $=0.009$ ). These results suggested that the risk of IAH increased almost linearly with the duration of glucocorticoid administration. On the other hand, administration of pulse glucocorticoid treatment was not significantly associated with IAH.

\section{Characteristics and fluid infusion differences observed in glucocorticoid and non-glucocorticoid-treated patients}

The clinical characteristics of patients in glucocorticoidand non-glucocorticoid-treated groups are compared in Tables S1 and S2. The baseline clinical severity (APACHEII score) was not significantly different (median 22 versus 19, $p=0.44$ ), while the levels of CRP and GCS on ICU admission were higher (median 9.62 versus $1.67 \mathrm{mg} / \mathrm{dL}, p=0.048$ and 15 versus $11, p=0.028$, respectively), and the levels of sodium and mean blood pressure were lower among glucocorticoid-related cases (median 139 versus $142 \mathrm{mEq} / \mathrm{L}$, $p=0.0018$ and 75.7 versus $98.7 \mathrm{mmHg}, p=0.025$, respectively). In comparison to the laboratory data on the day of IAH, the level of BUN was significantly higher among glucocorticoid-related cases (median 51 versus $33.5 \mathrm{mg} /$ $\mathrm{dL}, p=0.042$ ). The BUN/Cr ratio was also significantly higher among glucocorticoid-related cases $[39.6 \mathrm{mg} / \mathrm{dL}$ (IQR, 27.0-47.4) versus $27.0 \mathrm{mg} / \mathrm{dL}$ (IQR, 16.7-38.2), $p=0.0068]$.

Table 2 Factors associated with IAH

\begin{tabular}{|c|c|c|c|c|c|c|}
\hline & \multicolumn{2}{|l|}{ Univariate } & \multicolumn{2}{|c|}{ Multivariate model $1^{\mathrm{a}}$} & \multicolumn{2}{|c|}{ Multivariate model $2^{\mathrm{a}}$} \\
\hline & OR $(95 \% \mathrm{CI})$ & $p$ value & OR $(95 \%$ CI $)$ & $p$ value & OR $(95 \% \mathrm{CI})$ & $p$ value \\
\hline AKI & $2.22(1.36-3.63)$ & $0.002^{\mathrm{b}}$ & $2.76(1.39-5.47)$ & $0.004^{\mathrm{b}}$ & $2.72(1.31-5.62)$ & $0.007^{\mathrm{b}}$ \\
\hline Sepsis & $1.60(0.92-2.80)$ & 0.099 & $1.00(0.46-2.17)$ & 1.00 & $0.86(0.38-1.95)$ & 0.72 \\
\hline High-dose glucocorticoid ${ }^{c}$ & $2.38(1.04-5.43)$ & $0.040^{\mathrm{b}}$ & $3.52(1.20-10.3)$ & $0.022^{\mathrm{b}}$ & $4.15(1.29-13.4)$ & $0.017^{\mathrm{b}}$ \\
\hline Osmotic diuretics & $1.68(0.95-2.97)$ & 0.072 & $3.25(1.42-7.45)$ & $0.005^{\mathrm{b}}$ & $3.44(1.41-8.39)$ & $0.007^{\mathrm{b}}$ \\
\hline Loop diuretics & $1.63(0.92-2.89)$ & 0.093 & $1.28(0.61-2.72)$ & 0.52 & $1.41(0.63-3.15)$ & 0.40 \\
\hline Ventilation & $1.44(0.76-2.72)$ & 0.27 & $0.92(0.40-2.11)$ & 0.85 & $0.82(0.31-2.17)$ & 0.70 \\
\hline Renal replacement therapy & $0.47(0.19-1.14)$ & 0.096 & $0.20(0.062-0.64)$ & $0.007^{\mathrm{b}}$ & $0.20(0.052-0.75)$ & $0.017^{\mathrm{b}}$ \\
\hline Emergency surgery & $1.19(0.61-2.31)$ & 0.61 & $1.08(0.44-2.62)$ & 0.87 & $0.93(0.37-2.39)$ & 0.89 \\
\hline Infusion fluid, L & $1.23(1.02-1.48)$ & $0.030^{\mathrm{b}}$ & - & - & $1.11(0.75-1.64)$ & 0.60 \\
\hline Sodium, /10 mEq & $1.03(1.01-1.05)$ & $0.003^{\mathrm{b}}$ & - & - & $1.02(0.98-1.06)$ & 0.27 \\
\hline Glucose, /10 g & $1.00(0.98-1.04)$ & 0.59 & - & - & $1.01(0.95-1.08)$ & 0.73 \\
\hline Nitrogen, /g & $0.97(0.89-1.07)$ & 0.55 & - & - & $0.91(0.76-1.08)$ & 0.28 \\
\hline
\end{tabular}

GC glucocorticoid, CI confidence interval, APACHE Acute Physiology And Chronic Health Evaluation, AKI acute kidney injury

${ }^{a}$ Adjusted for age, gender, APACHEII score

${ }^{\mathrm{b}} p<0.05$

${ }^{\mathrm{c}}$ Including pulse administration 
Table 3 Influence of glucocorticoid administration on the risk of IAH

\begin{tabular}{|c|c|c|c|c|}
\hline & \multicolumn{2}{|l|}{ Univariate } & \multicolumn{2}{|l|}{ Multivariate $^{\mathrm{a}}$} \\
\hline & OR $(95 \%$ CI $)$ & $p$ value & OR (95\% CI) & $p$ value \\
\hline \multicolumn{5}{|l|}{ GC pulse administration $^{\mathrm{b}}$} \\
\hline With pulse & $0.97(0.29-3.25)$ & 0.965 & $0.79(0.17-3.66)$ & 0.76 \\
\hline Without pulse & $4.23(1.41-12.8)$ & $0.010^{\mathrm{c}}$ & $10.0(2.33-43.3)$ & $0.0020^{\mathrm{c}}$ \\
\hline Duration of GC in ICU & $1.17(1.00-1.36)$ & 0.051 & $1.21(1.01-1.45)$ & $0.039^{c}$ \\
\hline \multicolumn{5}{|l|}{ Duration (category) $^{\mathrm{b}}$} \\
\hline 1-3 days & $1.46(0.50-4.28)$ & 0.49 & $1.97(0.49-7.90)$ & 0.34 \\
\hline 4-6 days & $2.50(0.49-12.9)$ & 0.27 & $3.82(0.44-33.1)$ & 0.22 \\
\hline 7 days- & $8.79(1.07-72.0)$ & $0.043^{\mathrm{c}}$ & $14.1(1.35-147)$ & $0.027^{\mathrm{c}}$ \\
\hline Test for trend & & $0.015^{\mathrm{d}}$ & & $0.009^{\mathrm{d}}$ \\
\hline
\end{tabular}

$G C$ glucocorticoid, $C I$ confidence interval, APACHE Acute Physiology And Chronic Health Evaluation, $A K I$ acute kidney injury

${ }^{a}$ Adjusted for age, gender, APACHEII score, sepsis, AKI, use of osmotic diuretics, use of loop diuretics, use of high-dose glucocorticoid, use of mechanical ventilator, initiation of renal replacement therapy, and emergency operation prior to admission

${ }^{\mathrm{b}}$ Reference category is not administered GC

${ }^{c} p<0.05$

${ }^{\mathrm{d}} p$ values for trend tests examine whether increased duration of GC administration associate with increased odds ratios (duration categories were coded $0,1,2$, and for increasing the duration of GC administration)
The volume and contents of infusion fluid and urine volumes are shown in Fig. 1. Urine output in glucocorticoidrelated cases was not significantly different, but the total amounts of fluid infusion and sodium loads were significantly less than those in non-glucocorticoid-related cases. In glucocorticoid-related cases, the amounts of glucose and nitrogen were higher than in non-glucocorticoid-treated cases.

The median date of onset of glucocorticoid-related IAH cases was later than non- glucocorticoid-related IAH cases (median 9 versus 4 days, $p<0.001$ ). The duration of hypernatremia was longer in glucocorticoid-related IAH cases than non-glucocorticoid-related IAH cases (median 5 versus 2 days, $p=0.033$ ). The length of ICU stay was longer in glucocorticoid-related IAH cases than in non-glucocorticoidrelated IAH cases (median 13 versus 8 days, $p=0.0019$ ).

\section{Discussion}

This study, conducted in an established cohort of critically ill patients, found that high-dose glucocorticoid treatment was significantly associated with $\mathrm{IAH}$, more specifically there was a duration-response association with glucocorticoid administration and development of IAH. This study is the first to demonstrate the association between glucocorticoid and IAH by using a nested case-control study.

We compared the clinical characteristics, medical interventions, and complications of IAH cases and controls using a nested case-control study. This approach consisted of an observational study design using case-control matched subjects within an established cohort, which allows minimization of selection bias. This approach also allows the assessment of drug exposure that changes over time. Thus, we were able to evaluate the administration of glucocorticoids as a time-varying exposure of interest. In addition, aligning the index date with cases and controls allowed us to take into consideration the contents of the infusion fluid administered on the day before the onset of IAH, which was crucial for the investigation of electrolyte abnormalities. The ORs represent the unbiased estimates of incidence rate ratio from a person-time cohort study. In addition, the nested case-control design has the advantage of allowing statistically efficient analysis of data from a cohort with substantial savings of time and costs [20]. Thus, with this method, we could examine more detailed clinical parameters including medications and the contents of infusion fluid a day before IAH onset.

A previous study reported that the factors associated with hypernatremia were sepsis, hypokalemia, hypoalbuminemia, renal dysfunction, use of mannitol and use of sodium bicarbonate in a conventional case-control study design [2]. However, conventional case-control studies are prone to selection biases compared to the nested case-control design. Moreover, the authors did not consider glucocorticoid therapy as candidate risk factor of IAH. Darmon et al. reported that IAH was associated with male gender, greater disease severity at ICU admission, septic shock, acute respiratory failure, coma, and use of steroids [4]. Though the study suggested that the use of steroids was significantly associated with IAH, the authors did not 
A

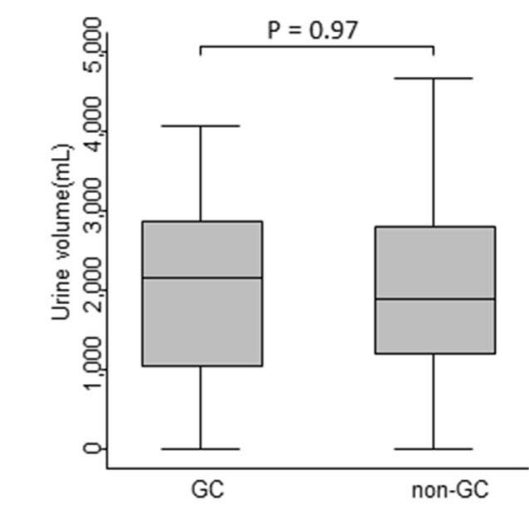

D

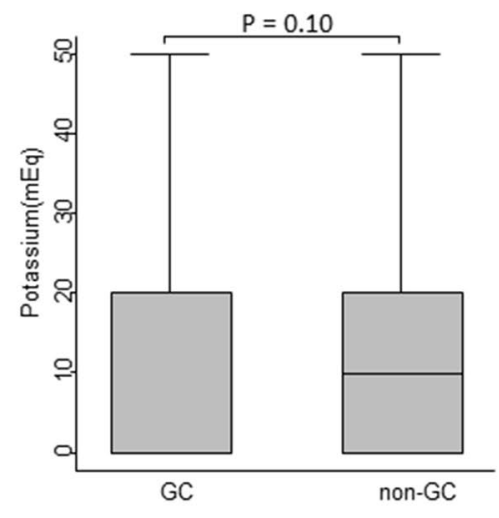

B

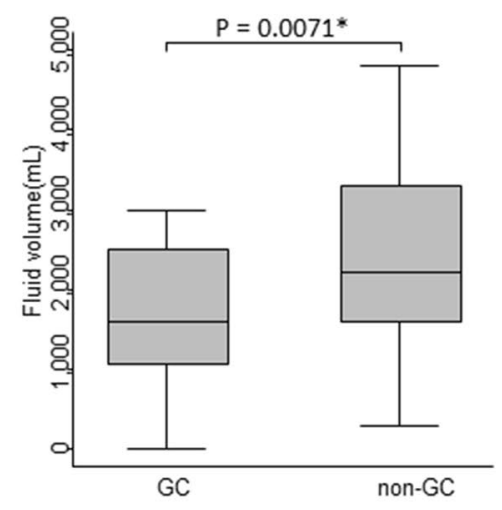

$\mathrm{E}$

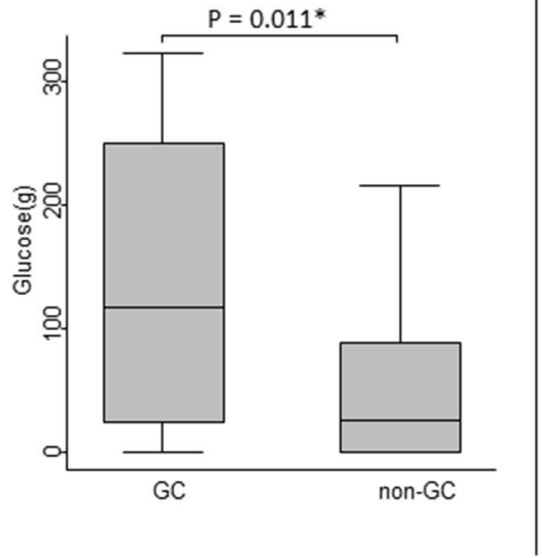

C

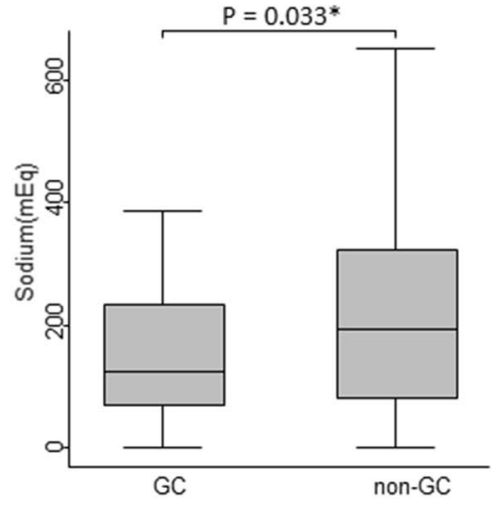

$\mathrm{F}$

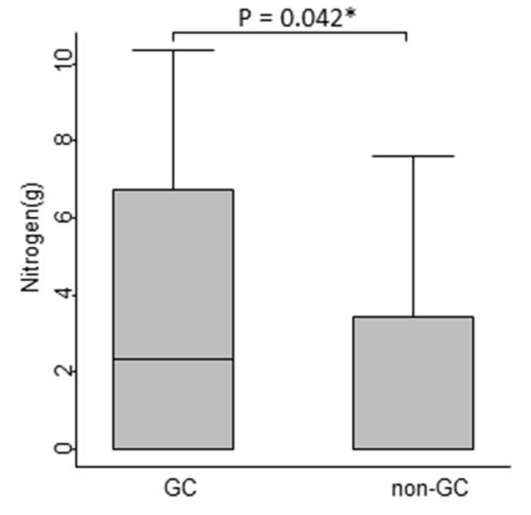

Fig. 1 Urine output and infusion fluid $24 \mathrm{~h}$ before the onset of IAH in glucocorticoid-related IAH cases and non-glucocorticoid-related IAH cases. a Urine volume (mL/day), b fluid volume (mL/day), c sodium

discuss the dose-response or duration-response effects, nor did they propose the mechanism involved.

Osmotic diuresis due to urea is an important cause of hypernatremia observed in the ICU [11]. Patients lose hypotonic fluid in the urine due to the osmotic urea diuresis induced by urea. At the same time, clinicians replaced these losses using isotonic fluids, which are hypertonic compared to the urine. Urea diuresis is a subtype of solute diuresis induced by non-electrolytes. Known causes include exogenous urea loading, protein or amino acid loading, hypercatabolic states, post-rhabdomyolysis, massive hematoma including gastrointestinal bleeding, diuretic phase of acute tubular necrosis, and post-obstructive diuresis [21]. In the present study, the levels of BUN and BUN/Cr were higher among glucocorticoid-related IAH cases, though osmotic diuresis due to urea could not be directly demonstrated because of lack of sufficient information about urinalysis. This may indicate that the
(mEq/day), d potassium (mEq/day), e Glucose (g/day), and $\mathbf{f}$ nitrogen (g/day). ${ }^{*} p<0.05$

glucocorticoid-related IAH cases were in a hypercatabolic state. In our results, the distribution of sodium intake was so wide that we could not completely deny the effect of sodium intake, although sodium intake was not significantly associated with IAH. However, we focused more on what we supposed that a hypercatabolic state could cause hypernatremia.

Glucocorticoid can induce hypernatremia not only by enhancing sodium retention, but also by increasing electrolyte-free water loss. It has been shown that patients, as well as experimental animals, exhibit polyuria in the presence of excess glucocorticoid hormones [22-24]. Experimental models have showed that excessive glucocorticoids leads to a urinary concentrating defect via downregulation of urea transporters, but not of aquaporin channels [25]. Most patients who need to be treated with glucocorticoids are in a hypercatabolic state characterized by respiratory failure and sepsis; glucocorticoid therapy may accentuate the 
pathological mechanism of urea diuresis in these patients and can cause hypernatremia as a consequence.

In terms of dose-response effects, use of pulsed glucocorticoid was not associated with IAH; rather, glucocorticoid without pulsed doses was significantly associated with IAH. There are two possible reasons for this. Firstly, pulse-administered glucocorticoid medications, such as methylprednisolone and dexamethasone, have relatively low mineralocorticoid activity, thus it may be unlikely that sodium retention occurred. Secondly, pulse administration may suppress inflammation earlier than high-dose glucocorticoid without pulse administration. On the other hand, longer duration of glucocorticoid exposure may maintain the systemic catabolic state longer and thus trigger ureainduced osmotic diuresis. Our results show that the longer you administer glucocorticoid, the higher is the possibility of developing IAH in the patient. From our results, the OR will increase by approximately $21 \%$ for each day the duration of glucocorticoid treatment is extended.

There are several limitations to this study. First, due to the retrospective nature of the study, there were numerous missing values in urine chemistry data. Furthermore, this was a single-center study, which can reduce the generalizability of the results. Urinary electrolytes could only be obtained in full from fewer than 20 patients of the cases group. We were thus unable to analyze the concentrations of urinary electrolytes fully. As a result, we could not estimate how many patients developed osmotic diuresis. Secondly, there is a possibility that we may have underestimated the number of IAH patients because we obtained the data only from those patients who remained in the ICU for more than 2 days. Whatever treatment patients had received in ICU, once they were discharged from the ICU, we were unable to determine whether they developed hypernatremia. Thus, we may have missed those patients exhibiting hypernatremia after being discharged from the ICU. Misclassification of potential IAH cases can be yielded in a different mechanism. Study participants were only decided by whether the maximum sodium concentration exceeded $150 \mathrm{mEq} / \mathrm{L}$ during the ICU stay. Even if the peak sodium concentrations were within the range below 149, some patients would have had larger changes in concentration. Since glucocorticoid-related hypernatremia developed at a relatively later phase in the ICU in this study, this misclassification may attenuate the true effect of GC on developing IAH, which can enhance the true effect and support robust conclusion (i.e., bias toward the null hypothesis). Third, this study adopted high-dose glucocorticoid as $>30 \mathrm{mg}$ prednisone equivalent per day regardless of patient body mass. This may have misclassified underweight individuals (e.g., those who were taking moderate dose of steroid, but less than $30 \mathrm{mg}$ prednisone equivalent per day) into the GC naive group. However, we considered that this was an undifferenciated misclassification, since the median body mass index in the both groups was not significantly different. Fourth, our results showed that renal replacement therapy could reduce the possibility of IAH. This study design did not allow us to exclude patients who underwent renal replacement therapy because they were not necessarily done at baseline. Finally, the case-control study design is susceptible to biases; however, we have minimized potential biases by using a nested case-control design .

\section{Conclusions}

In the present study, we clearly demonstrated that high-dose glucocorticoid is strongly associated with IAH. When we administer high-dose glucocorticoid to critically ill patients, close monitoring of their serum sodium concentrations should be required especially when glucocorticoid is administered for longer periods.

Acknowledgements The authors acknowledge the physicians and patients of Toyohashi Municipal Hospital, who contributed to this study. The authors also acknowledge Ms. Megumi Matsushita (medical assistant) for extracting the electrical data and the medical records of all patients, and Mr. Shozo Nakamura (medical engineer), Mr. Shin'ichi Miura (medical engineer), and Mr. Hironori Aoyama (medical engineer) for providing the lists of patients who underwent mechanical ventilation and renal replacement therapy.

\section{Compliance with ethical standards}

Conflict of interest The authors have declared that no conflict of interest exists.

Ethical approval All procedures performed in studies involving human participants were in accordance with the ethical standards of the institutional research committee at which the studies were conducted (IRB approval number 248) and with the 1964 Helsinki Declaration and its later amendments or comparable ethical standards.

Informed consent Informed consent was not obtained since this was a non-invasive retrospective observational study.

\section{References}

1. Lindner G, Funk GC, Schwarz C, et al. Hypernatremia in the critically ill is an independent risk factor for mortality. Am J Kidney Dis. 2007;50:952-7.

2. Hoorn EJ, Betjes MGH, Weigel J, Zietse R. Hypernatraemia in critically ill patients: too little water and too much salt. Nephrol Dial Transplant. 2008;23:1562-8.

3. O'Donoghue SD, Dulhunty JM, Bandeshe HK, Senthuran S, Gowardman JR. Acquired hypernatraemia is an independent predictor of mortality in critically ill patients. Anaesthesia. 2009;64:514-20.

4. Darmon M, Timsit JF, Francais A, et al. Association between hypernatraemia acquired in the ICU and mortality: a cohort study. Nephrol Dial Transplant. 2010;25:2510-5. 
5. Waite MD, Fuhrman SA, Badawi O, Zuckerman IH, Franey CS. Intensive care unit-acquired hypernatremia is an independent predictor of increased mortality and length of stay. J Crit Care. 2013;28:405-12.

6. Kozeny GA, Murdock DK, Euler DE, et al. In vivo effects of acute changes in osmolality and sodium concentration on myocardial contractility. Am Heart J. 1985;109:290-6.

7. Lenz K, Gössinger H, Laggner A, Druml W, Grimm G, Schneeweiss B. Influence of hypernatremic-hyperosmolar state on hemodynamics of patients with normal and depressed myocardial function. Crit Care Med. 1986;14:913-4.

8. Knochel JP. Neuromuscular manifestations of electrolyte disorders. Am J Med. 1982;72:521-35.

9. Polderman KH, Schreuder WO, Strack van Schijndel RJ, Thijs LG. Hypernatremia in the intensive care unit: an indicator of quality of care? Crit Care Med. 1999;27:1105-8.

10. Sam R, Feizi I. Understanding hypernatremia. Am J Nephrol. 2012;36:97-104.

11. Lindner G, Schwarz C, Funk GC. Osmotic diuresis due to urea as the cause of hypernatraemia in critically ill patients. Nephrol Dial Transplant. 2012;27:962-7.

12. Annane D, Bellissant E, Bollaert PE, Briegel J, Keh D, Kupfer Y. Corticosteroids for treating sepsis. Cochrane database Syst Rev. 2015;12:CD002243.

13. Imaizumi $T$, Nakatochi M, Fujita $Y$, et al. The association between intensive care unit-acquired hypernatraemia and mortality in critically ill patients with cerebrovascular diseases: a single-centre cohort study in Japan. BMJ Open. 2017;7:e016248.

14. Buttgereit F, da Silva JAP, Boers M, et al. Standardised nomenclature for glucocorticoid dosages and glucocorticoid treatment regimens: current questions and tentative answers in rheumatology. Ann Rheum Dis. 2002;61:718-22.

15. Lubin JH, Gail MH. Biased selection of controls for case-control analyses of cohort studies. Biometrics. 1984;40:63-75.
16. Clayton DGHM. Analysis of follow up studies with Stata 5.0. Stata Tech Bull. 1997;40:12-8.

17. Knaus WA, Draper EA, Wagner DP, Zimmerman JE. APACHE II: a severity of disease classification system. Crit Care Med. 1985;13:818-29.

18. Singer M, Deutschman CS, Seymour CW, et al. The third international consensus definitions for sepsis and septic shock (sepsis-3). JAMA. 2016;315:801-10.

19. Kellum Ja, Lameire N, Aspelin P, et al. KDIGO clinical practice guideline for acute kidney injury. Kidney Int Suppl. 2012;2:1-138.

20. Essebag V, Genest J, Suissa S, Pilote L. The nested case-control study in cardiology. Am Heart J. 2003;146:581-90.

21. Oster JR, Singer I, Thatte L, Grant-Taylor I, Diego JM. The polyuria of solute diuresis. Arch Intern Med. 1997;157:721-9.

22. Thunhorst RL, Beltz TG, Johnson AK. Glucocorticoids increase salt appetite by promoting water and sodium excretion. Am J Physiol Regul Integr Comp Physiol. 2007;293:R1444-51.

23. Kalimi M. Role of antiglucocorticoid RU 486 on dexamethasone-induced hypertension in rats. Am J Physiol. 1989;256:E682-E685685.

24. Ferrari P. Cortisol and the renal handling of electrolytes: role in glucocorticoid-induced hypertension and bone disease. Best Pract Res Clin Endocrinol Metab. 2003;17:575-89.

25. Li C, Wang W, Summer SN, Falk S, Schrier RW. Downregulation of UT-A1/UT-A3 is associated with urinary concentrating defect in glucocorticoid-excess state. J Am Soc Nephrol. 2008;19:1975-81.

Publisher's Note Springer Nature remains neutral with regard to jurisdictional claims in published maps and institutional affiliations. 